Dom. Cien., ISSN: 2477-8818

Vol. 5, núm.1., ene, 2019, pp. 385-400

Incentivo del liderazgo compartido en la Educación Superior

\title{
Incentivo del liderazgo compartido en la Educación Superior
}

\author{
Incentive of shared leadership in higher education
}

\section{Incentivo à liderança compartilhada no ensino superior}

Miguel Á. Lombeida-Carballo I

miguelomcvd@yahoo.es

Recibido: 10 de octubre de 2018 * Corregido: 30 de octubre de 2018 * Aceptado: 30 de noviembre de 2018

I. Master en Pedagogía Profesional; Licenciado en Ciencias de la Educación Profesor de Enseñanza Media en la Especialización de Educación Física; Universidad Estatal de Bolívar, Guaranda, Ecuador. 


\section{Resumen}

Los nuevos retos en la educación superior de hoy requieren nuevas formas de liderazgo. Un ambiente financiero muy volátil, el incremento de las asociaciones corporativas internacionales, mayores presiones en la rendición de cuentas, la necesidad de nuevos modelos de negocio, las nuevas tecnologías y los cambios demográficos son solo algunos de estos desafíos, que requieren soluciones de liderazgo que se prueban tanto dentro como fuera de la educación superior. El liderazgo compartido consistentemente surge como un factor clave para las organizaciones que tienen mayor capacidad de aprender, innovar, actuar y adaptarse a los tipos de desafíos externos que los campus ahora enfrentan. El liderazgo compartido se define como alejarse de la figura líder / seguidor; capitalizando la importancia de líderes en toda la organización, no solo los que ocupan cargos de autoridad; y creando una infraestructura para que las organizaciones puedan beneficiarse del liderazgo de múltiples personas.

Este informe examina cómo un contexto ambiental cambiante en la educación superior requiere un nuevo liderazgo, habilidades y enfoques, siendo el principal de ellos los principios del liderazgo compartido. Se revisa el nuevo ambiente de liderazgo, la investigación sobre liderazgo compartido y el pequeño cuerpo de investigación en educación superior.

Palabras claves: Liderazgo; Educación Superior; Innovación; Nuevas Tendencias. 


\begin{abstract}
The new challenges in higher education today require new forms of leadership. A very volatile financial environment, the increase in international corporate partnerships, greater pressures in accountability, the need for new business models, new technologies and demographic changes are just some of these challenges, which require leadership solutions that they are tested both inside and outside of higher education. Shared leadership consistently emerges as a key factor for organizations that are better able to learn, innovate, act and adapt to the types of external challenges that campuses now face. Shared leadership is defined as moving away from the leader / follower figure; capitalizing on the importance of leaders throughout the organization, not just those in positions of authority; and creating an infrastructure so that organizations can benefit from the leadership of multiple people.
\end{abstract}

Key words: Leadership; Higher education; Innovation; New tendencies. 


\section{Introducción.}

Los nuevos modelos de liderazgo reconocen que la efectividad en entornos basados en el conocimiento depende menos en las acciones heroicas de unos pocos individuos en la parte superior y más en las prácticas de liderazgo colaborativo distribuido en toda la organización, lo que sugiere que un concepto relacional más dinámico del liderazgo ha emergido (Pearce \& Conger, 2003). La cita anterior capta cómo los complejos entornos de hoy requieren nuevas formas de colaboración o liderazgo colaborativo para ayudar a los campus a ser más ágiles y receptivos a los cambios necesarios del mundo de hoy. Los líderes de la educación superior ahora se enfrentan a un conjunto muy diferente de desafíos que requieren nuevas formas de liderazgo: por ejemplo, la volatilidad del entorno financiero, el auge de las alianzas mundiales e internacionales, mayores presiones de rendición de cuentas en torno a la finalización de la universidad y los resultados de aprendizaje, la necesidad de nuevos modelos de negocios, oportunidades para innovación con tecnología y cambios demográficos, mientras que la educación superior ha sufrido períodos de cambios significativos, particularmente después de la segunda guerra mundial, cuando las inscripciones crecieron significativamente, el entorno de hoy es único en términos de la gran cantidad de áreas que exigen cambios. Además, la mayoría de los comentaristas sugieren que los enfoques actuales de liderazgo no son efectivos para administrar el alcance de estos cambios.

Dada esta era actual de cambios significativos en la educación superior, hay una creciente atención a la importancia de entender el liderazgo requerido para guiar a los campus con éxito y una creciente preocupación que los enfoques existentes para el liderazgo son ineficaces. Históricamente, el tipo de liderazgo requerido para el cambio en la empresa ha cambiado. Algunas veces ha sido promulgada en gran parte por los presidentes, otras veces en consulta y la colaboración con el 
profesorado, y más recientemente (aunque no exclusivamente) ha habido de nuevo más esfuerzos de arriba hacia abajo por parte de los presidentes para gestionar el cambio en el campus. Desafortunadamente, el impulso actual para un mayor liderazgo de arriba hacia abajo es contraproducente para el panorama de la educación superior de hoy y está en desalineación con investigaciones sobre organizaciones efectivas que demuestran la necesidad de un liderazgo compartido.

Si bien las partes interesadas y los defensores dirán que el verdadero cambio requiere un liderazgo de arriba hacia abajo, también es el caso de que el cambio requiera un liderazgo compartido. La investigación también demuestra que las organizaciones necesitan una mayor capacidad para aprender y adaptarse en este entorno complejo, que también requiere un tipo de liderazgo diferente al del pasado (Alvarado, Prieto, \& Betancourt, 2009).

En la década de 1980, los investigadores identificaron cómo las compañías japonesas superaron a las estadounidenses. Las empresas debido a su capacidad para innovar y cambiar de manera flexible mediante el uso de la gestión de la calidad en sus procesos; por ejemplo, al delegar autoridad a los empleados para realizar cambios, creando una cultura que apoyó la toma de riesgos y el trabajo en equipos multifuncionales para gestionar los procesos de trabajo de manera más holística (Kezar A. J., 2001). En los años siguientes, los investigadores de los Estados Unidos exploraron estos principios en el contexto de las empresas americanas. Los estudios de investigación realizados durante las siguientes décadas identificaron claramente las prácticas que hacen que las organizaciones sean más adaptables y el tipo de liderazgo que apoya la innovación: liderazgo compartido. De hecho, el liderazgo compartido emergió consistentemente como un factor clave para las organizaciones que fueron más capaces de aprender, innovar y actuar. 
Entre otros atributos, muchos argumentan que el liderazgo compartido podría hacer que la educación superior sea más responsable a las partes interesadas externas, ya que el liderazgo compartido permite a las instituciones crear oportunidades significativas y cambios duraderos en las organizaciones que abordan los desafíos externos. Edificaciones de liderazgo compartido memoria institucional y crea una copropiedad sobre los objetivos y estrategias aspiracionales que de otro modo podrían desaparecer con la facturación ejecutiva. Todos los estudios están de acuerdo en que la rapidez social, política, económica y los cambios tecnológicos que se están produciendo están produciendo una mayor complejidad y un aumento de la inestabilidad, que imponen importantes limitaciones a los constructos convencionales de liderazgo de arriba hacia abajo.

Un informe reciente de ACE (American Council on Education) sobre modelos de negocios en educación superior en evolución: liderando con datos para entregar resultados: también demuestra que los campus necesitan más formas de liderazgo compartidas y en red para la toma de decisiones de presupuesto para abordar la creciente complejidad. En resumen, los líderes del campus enfrentan el desafío de implementar más cambios que nunca, en un cambiante paisaje social, político y económico, conformado por la complejidad. Enfoques compartidos para el liderazgo que capitalizar el conocimiento más amplio de la institución y fomentar el aprendizaje son necesarios para avanzar. Si bien muchos campus piensan que fomentan el liderazgo compartido a través de mecanismos como la gobernanza compartida, en este trabajo se demuestra cómo muchos campus son en general lamentablemente inadecuados para apoyar el verdadero liderazgo compartido.

\section{Metodología.}

La revisión se ha centrado en textos, documentos y artículos científicos publicados disponibles en la web, considerando que aquella herencia de la globalización nos permite acceder a 
mayor y mejor información a través de las herramientas tecnológicas. Los criterios de inclusión se basaron en publicaciones del habla hispana e inglesa y visible en bases de datos que nos aportaron en la historia y evolución de investigación. El motor de búsqueda ha sido herramientas académicas de la web que direccionan específicamente a archivos con validez y reconocimiento científico, descartando toda información no confirmada o sin las respectivas referencias bibliográficas.

\section{Resultados.}

Si bien hay varias definiciones de liderazgo compartido, todas comparten algunas características comunes:

1. Un mayor número de individuos en el liderazgo que los modelos tradicionales.

2. Los líderes y seguidores son vistos como intercambiables.

3. El liderazgo no se basa en la posición o la autoridad.

4. Las múltiples perspectivas y la experiencia se capitalizan para la resolución de problemas, la innovación y el cambio.

5. Se enfatiza la colaboración y las interacciones en toda la organización.

Las formas compartidas de liderazgo prescinden de la idea de un líder / seguidor binario, maximizando las contribuciones entre muchas más personas que pueden resolver problemas difíciles. El liderazgo compartido también reconoce la importancia de los líderes en posiciones de autoridad, pero se enfoca en cómo aquellos en posiciones de poder pueden delegar autoridad, capitalizar la experiencia dentro de la organización, y crear infraestructura para que las organizaciones puedan capitalizar el liderazgo de múltiples personas. El liderazgo es un proceso, no 
un individuo, y puede ser apoyado por el desarrollo profesional, acceso a la información, trabajo en equipo e incentivos.

Por ejemplo, los marcos de liderazgo de complejidad demuestran que la gestión científica tradicional y los principios de liderazgo (burocracia, autoridad, conductas de liderazgo predecibles y control social) son estrategias fallidas en tiempos de turbulencia ambiental (Allen \& Cherrey, 2000).En marcado contraste con estos puntos de vista tradicionales, los teóricos del liderazgo de complejidad reconocen lo ambiguo, realidades múltiples y siempre cambiantes de las organizaciones que operan dentro de las sociedades globales modernas. Ellos en lugar de avanzar un marco de liderazgo que postule el logro de una estabilidad global a nivel de sistema a través de apoyo a la autonomía, flexibilidad, creatividad y adaptabilidad a nivel local, la aplicación de estrictas reglas organizativas aplicadas sin consideración de contexto, mecanismos centralizados de toma de decisiones, y la diferenciación de tareas asociadas con la jerarquía organizacional (todas las características del liderazgo tradicional)sirven para cementar estructuras y prácticas incapaces de responder a las constantes fluctuaciones y cambio de prioridades que caracterizan a las organizaciones caóticas y complejas.

Para prosperar en medio de la complejidad, las organizaciones deben adoptar procesos organizativos que prioricen la colaboración, el liderazgo compartido y la toma de decisiones locales. La descentralización y la promoción de autonomía local aumenta la adaptabilidad de las organizaciones y les permite de forma creativa y rápida responder a las condiciones ambientales cambiantes (Adan, 2006).

En complejidad y sistema, las teorías de liderazgo, el equipo y los procesos de liderazgo colaborativo desafían a las organizaciones a mirar más allá las habilidades y logros individuales y, 
en cambio, centran su energía en cultivar entornos que enfaticen las interconexiones, una visión compartida para el futuro y los logros colectivos.

El liderazgo compartido, la colaboración y la creatividad también son componentes críticos del liderazgo adaptable (Heifetz, 1994). Heifetz critica los modelos tradicionales de liderazgo por su preocupación por resolver la rutina, problemas técnicos en lugar de movilizar esfuerzos de liderazgo para enfrentar los desafíos complejos y de adaptación que enfrentan las organizaciones globales que operan en un estado de flujo constante. Heifetz describe un modelo de adaptación de liderazgo que abarca la complejidad y la ambigüedad y busca activamente soluciones innovadoras a través de la organización, aprendizaje, resolución creativa de problemas, experimentos y colaboración. La educación superior necesita responder mejor a las presiones externas para el cambio, y la investigación sobre liderazgo compartido que sugiere permitir a los campus crear cambios que sean sostenibles.

El liderazgo compartido también se considera complementario a los modelos de contingencia y situación a largo plazo de liderazgo. Porque el liderazgo compartido puede capitalizar los variados rasgos de liderazgo, comportamientos, los estilos y procesos se consideran más adaptables a diferentes situaciones y contextos. Modelos de contingencia de liderazgo han sugerido durante mucho tiempo que los líderes no pueden usar los mismos comportamientos o enfoques en diferentes situaciones. Una crisis frente a un proceso de cambio más continuo utilizará y requerirá diferentes formas de liderazgo para ser exitoso. 
A nivel conceptual, la noción de liderazgo compartido parece estar bien alineada con las nociones de colegialidad y la autonomía profesional que tradicionalmente han sido características del liderazgo en la educación superior, al tiempo que reconoce las necesidades institucionales más amplias para gestionar eficazmente los retos que los entornos turbulentos imponen a las instituciones de educación superior.

Gran parte de la literatura sobre liderazgo compartido en la educación superior ha sido descriptiva, con muchos argumentos por los beneficios del liderazgo compartido extraídos de los estudios descritos anteriormente. Sin embargo, existe un pequeño cuerpo de investigación empírica que respalda el valor, los beneficios y el diseño del liderazgo compartido dentro de la educación superior. El trabajo seminal de (Birnbaum, 1992) fue uno de los primeros estudios en aludir a la importancia del liderazgo compartido en la identificación delas limitaciones del liderazgo presidencial, en particular. Después de cinco años de estudiar la presidencia, Robert Birnbaum determinó que los equipos y los gabinetes eran esenciales para el liderazgo del campus y que ese liderazgo individual no proporcionó la complejidad cognitiva y la experiencia necesaria para administrar la universidad de manera efectiva. Birnbaum afirmó: "cuando se comparte el liderazgo, una universidad tiene múltiples formas de detectar el cambio del medio ambiente, comprobación de problemas y supervisión del rendimiento del campus. Con el liderazgo compartido es probable proporcionar a una universidad una forma de pensar más compleja". El estudio examinó la complejidad cognitiva para la toma de decisiones entre los presidentes y encontró que pocos utilizaban el tipo de pensamiento de múltiples marcos, eso está alineado con una mejor toma de decisiones. Esta investigación sugiere que los presidentes deben trabajar con otros para ser efectivos y que los líderes de marcos múltiples eran relativamente raros. Los líderes tienden a analizar 
situaciones de manera simplista utilizando solo uno o dos marcos organizacionales o modelos mentales.

Si bien el estudio identificó la forma en que el liderazgo compartido crea una mayor complejidad cognitiva e innovación, también identificó otros resultados. Los procesos de liderazgo del equipo crean vehículos de apoyo entre iguales en tiempos de desafío y crisis y sirven para aumentar la responsabilidad dentro de las organizaciones, dado que en el equipo los miembros pueden responsabilizarse mutuamente de llevar a cabo los roles y responsabilidades designados.

El liderazgo puede ser un paso esencial hacia el cultivo de entornos organizativos inclusivos que aprovechen las perspectivas y experiencias únicas de grupos sociales históricamente marginados, además de mejorar la implementación de las decisiones organizativas ya que los miembros dedican tiempo y energía por adelantado para fomentar una visión compartida y la apropiación colectiva en las acciones organizativas.

Si bien existe poca investigación empírica sobre los resultados del liderazgo compartido en la educación superior, hay un poco más de trabajo sobre los antecedentes o condiciones que promueven su desarrollo. Estos se alinean con la base de investigación multidisciplinaria e incluye apoyo de líderes verticales, recursos, desarrollo de apoyo a culturas a través de la construcción de relaciones y el desarrollo profesional, clara definición de roles, autonomía, objetivos compartidos, experiencia interna clave, retroalimentación externa, intercambio de información, estructuras de rendición de cuentas, y la inclusión.

Para obtener los beneficios del liderazgo compartido, las organizaciones deben garantizar que las estructuras y procesos sean auténticos y cuidadosamente diseñados. Por ejemplo,(Tablada, 
2008) noto la ausencia de un liderazgo auténticamente compartido en los departamentos y escuelas de la mayoría de las universidades que estudio. En cambio, el liderazgo compartido era principalmente una estrategia retórica utilizada por los líderes verticales para dar la impresión de inclusión y colaboración, sin prestar atención a la definición clara de roles o la diferenciación de tareas, el liderazgo compartido puede convertirse en conflicto e incertidumbre.

Además de los desafíos con el diseño y la autenticidad del liderazgo compartido, es importante comprender algunas críticas del liderazgo compartido para navegar los problemas que puedan surgir. La crítica más prominente al liderazgo compartido es que a menudo no aborda temas muy reales de conflicto, poder y autoridad. A medida que más partes interesadas son involucradas en la toma de decisiones, los diferentes intereses y la política tienen más probabilidades de surgir. Los miedos y, a veces, las realidades de los diferentes intereses y valores que desaceleraron dramáticamente el liderazgo ha llevado a muchos administradores del campus a evitar incluir una gama más amplia de personas en un liderazgo compartido auténticamente. De hecho, estos temores han llevado a una disminución en los aportes de los grupos de partes interesadas en la educación superior en los últimos años (Kezar \& Eckel, 2004)

El liderazgo compartido se entiende o estudia mejor con una apreciación de los valores organizacionales de la educación superior, como la gobernanza compartida, autonomía institucional y profesional y libertad académica. Muchas de estas características que han definido la educación superior a menudo impiden compartir el liderazgo, aunque puedan parecer complementarios a primera vista. Como se señaló anteriormente, la gobernanza compartida (así como la libertad académica y la autonomía) se centra en la distribución de la autoridad en lugar de colaboración. Y, sin embargo, es la colaboración la clave para la creatividad y la complejidad cognitiva. La 
autonomía y la libertad académica también se basan en principios que los profesionales como expertos tienen autoridad delegada, pero a menudo no hay estructuras claras de rendición de cuentas por esa autoridad. El liderazgo compartido se enfoca en establecer estructuras de responsabilidad distribuida. Así, Una de las razones por las que los campus suelen tener dificultades para establecer un liderazgo compartido es que, si bien es en relación con estas estructuras históricas y complementarias, también difiere en aspectos fundamentales.

Una preocupación final relacionada con el liderazgo compartido es que las personas que trabajan juntas, particularmente en equipos, pueden desarrollar pensamiento grupal. El pensamiento grupal, originalmente conceptualizado por (Janis, 1982), se caracteriza poruna especie de "búsqueda extrema de consenso" en la que los puntos de vista alternativos se anulan, la crítica se convierte en imposible, y malos resultados en la toma de decisiones. Si bien esta preocupación es legítima, muchos estudios han encontrado que la cohesión del equipo en general no conduce al pensamiento grupal y, en cambio, generalmente facilita las relaciones, interacciones y el rendimiento de los grupos. Adicionalmente, Si el liderazgo compartido se desarrolla para capitalizar verdaderamente una amplia gama de habilidades y experiencias, como lo está destinado a hacer, el pensamiento grupal se vuelve menos probable.

\section{Conclusiones.}

La mayoría de los programas de desarrollo de liderazgo tienden a centrarse en personas que ya están (o aspiran a estar) en posiciones de autoridad, a menudo con líderes individuales en lugar de equipos, además los programas tradicionales de desarrollo de liderazgo tienden a centrarse en rasgos, habilidades o comportamientos que ayudan a un individuo en una posición de autoridad para promulgar el liderazgo. Los programas orientados a estos rasgos intentan identificar y cultivar 
características personales específicas, tales como integridad, compromiso, inteligencia y confianza, que contribuyen a la capacidad de una persona para asumir y funcionar con éxito en posiciones de liderazgo. Probablemente el modelo de liderazgo más común se centra en el desarrollo de habilidades y tareas asociadas con el liderazgo, como la planificación, la recaudación de fondos, o negociación. Tanto las perspectivas de liderazgo basadas en rasgos como en habilidades dependen únicamente de los líderes individuales para desarrollar liderazgo.

En cambio, los programas deben ser rediseñados para atraer equipos de liderazgo y deben centrarse en más que solo habilidades para la promulgación individual. Tales programas deberían ayudar a los equipos a trabajar juntos para crear planes estratégicos o un entorno más amplio de confianza. Además, cuando reconocer el liderazgo es un proceso, y no un apersona, la gente puede ser entrenada en formas para facilitar ese proceso. Este entrenamiento debe incluir atención a Contexto institucional y cultura para que los líderes puedan crear mejor un proceso que sea efectivo para su entorno. Con este cambio de perspectiva en mente, los modelos de liderazgo compartido subrayan la necesidad de programas de desarrollo de liderazgo para cambiar su enfoque exclusivo de la identificación y el cultivo del liderazgo individual.

El desarrollo del liderazgo en la educación superior tal como está diseñado actualmente no es efectivo para fomentar liderazgo. Somos conscientes de muy pocos programas que ayudan a los líderes a crear entornos de liderazgo compartido. Kezar proporcionan consejos detallados para los líderes sobre cómo crear un entorno de liderazgo compartido; Este trabajo podría usarse para diseñar o mejorar programas de liderazgo, junto con Kezar, que describe los elementos de los programas de desarrollo de liderazgo que facilitan el liderazgo compartido alineado con las necesidades actuales. 
¿Cómo pueden los líderes existentes en la educación superior promover formas de liderazgo más colaborativas y compartidas en sus campus? Los líderes deben comenzar por identificar desafíos complejos en sus instituciones: problemas espinosos que no han sido bien atendidos por las estrategias tradicionales o existentes. Ejemplos de algunos de estos temas complejos se han intercalado a lo largo de este documento, pero también podrían incluir áreas como eliminando cursos que resultan en un alto desgaste, apoyando el éxito de transferencia de estudiantes, implementando nuevos mecanismos de evaluación o la creación de nuevos programas académicos para satisfacer una necesidad de la comunidad. Una vez se han identificado tales desafíos, se reúne a un equipo multifuncional de líderes de todo el campus. Al crear un equipo de este tipo, se debe buscar cómo incorporar el conocimiento de toda la institución, para ejemplo, facultad, asuntos estudiantiles, investigación institucional, registrador, biblioteca, centros de enseñanza y aprendizaje, instalaciones, y más. Existen potenciales reservorios de experiencia sin explotar en lugares inesperados que podrían ayudar a resolver problemas.

Para que los colegios y universidades puedan realmente cosechar los beneficios de formas de liderazgo más colaborativas, las instituciones que toman las decisiones deben estar dispuestos a reexaminar cuidadosamente sus propias concepciones de lo que significa ser un líder exitoso. Si un presidente o rector sigue pensando en el liderazgo como una búsqueda heroica solitaria, cualquier esfuerzo por establecer estructuras de liderazgo compartido no creará cambios significativos. Los líderes del campus tienen derecho a acoplar su apoyo externo por esfuerzos de liderazgo compartido con reflexión interna sobre cómo el liderazgo puede y debe funcionar de una manera cada vez más compleja dentro del sistema educativo superior. Si los líderes están dispuestos a experimentar con estos nuevos enfoques, sus instituciones están preparadas para enfrentar estas complejidades y desafíos de frente. 


\section{Bibliografía.}

Adan, E. (2006). Liderazgo renovador y esperanzador para construir la universidad del futuro. Laurus, 12(1), 170-179.

Allen, K. E., \& Cherrey, C. (2000). Liderazgo Sistematico. Bogotá: UMNG.

Alvarado, Y., Prieto, A., \& Betancourt, D. (2009). Liderazgo y motivacion en el ambiente educativo universitario. ctualidades Investigativas en Educación, 9(3), 1-18.

Birnbaum, R. (1992). Como funciona el liderazgo academico.

Heifetz, R. A. (1994). Leadership Without Easy Answers.

Janis, I. L. (1982). Groupthink: Psychological Studies of Policy Decisions and Fiascoes.

Kezar, A. J. (2001). Understanding and Facilitating Organizational Change in the 21 st Century. ERIC, 28(4).

Kezar, A. J., \& Eckel, P. (2004). "Meeting Today's Governance Challenges: A Synthesis of the Literature and Examination of a Future Agenda for Scholarship."

Pearce, C. L., \& Conger, A. (2003). Shared Leadership: Reframing the Hows and Whys of Leadership.

Tablada, J. (2008). El liderazgo educativo debe ser un liderazgo transformador 